\title{
Theoretical Characterization of the Long-range Attraction between G-actin Molecules through the Excluded Volume Effect
}

\author{
M. IRISA \\ Department of Biochemical engineering and Science, Faculty of Computer Science and Systems \\ Engineering, Kyushu Institute of Technology, 680-4 Kawazu, Iizuka-city, Fukuoka 820-8502, Japan \\ (E-mail: irisa@bse.kyutech.ac.jp)
}

\begin{abstract}
One of the interactions between macromolecules is the attractive force through the excluded volume effect. We studied the attraction between the molecules of muscle protein, actin, in the two points by using the extended scaled particle theory (XSPT). I) we verified the basic assumption used in the XSPT that topological elements which determine the analytical expression of the excluded volume are almost unchanged through the scaling down of the solute molecule in the thought experiment. Results of the computational geometry method ( $\alpha$-shape method) showed that this assumption is valid even in the case of the actin molecule. II) we calculated the attraction between actin monomer molecules, G-actin. Calculated differences of the values of the attraction potential of two macromolecules between at contact and at one macromolecule apart by the XSPT is almost the same as those by the Asakura-Oosawa theory.
\end{abstract}

Key words: actin, a-shape, association, excluded volume, muscle protein, scaled particle theory

\section{Introduction}

Under the biological condition, the muscle protein G-actin monomer forms the filament, F-actin, and 3-D structure of the monomer in the F-actin is changed [1]. It is thought that the association of G-actin occurres in the first stage of the filament formation. Generally, the intermolecular interaction, especially long-range attraction, between macromolecules affects the association. The attraction between the biomolecules is intensively studied to search not only the filament formation but also the function, e.g. force generation of the muscle protein. One of the interactions between macromolecules is the attraction through the excluded volume effect. Asakura-Oosawa proposed the simple equation (AO theory) to estimate the attraction by considering the geometry of the excluded volume of the macromolecules [2]. Their theory is well agreed with the experimental results in spite of the usage of rough assumptions [3-5]. In the AO theory, the intermolecular force is derived from the geometric consideration of the excluded volume of the macromolecule and the microscopic osmotic pressure at the molecular size. On the other hand, we 
recently extended the widely used solution theory, scaled particle theory (SPT) [6], to the arbitrary shaped solute molecule [7, 8]. The extended SPT (XSPT) has been used in the calculation of the solvation free energy of the protein molecule [9]. The basis of the XSPT is the theoretical derivation of the microscopic pressure on the solute molecule by using the statistical mechanics.

In the case of the interaction of two macromolecules in the dilute macromolecular solution, the excluded volume of the two macromolecules can be regarded as the cavity where the center of the surrounding macromolecule cannot enter. Then the attractive force through the excluded volume effect can be interpreted as the minimization process of the work required to make the cavity in the macromolecular solution. Normally in the XSPT, the contribution from the repulsive force between the solute and the solvent is calculated as the work to make the cavity in the solvent. When the XSPT is applied to the macromolecular solution, it is assumed that the degrees of freedom of the solvent molecule are neglected by following the traditional polymer theory. The pressure applied to the macromolecules in XSPT corresponds to the osmotic pressure of the macromolecules.

We found that the XSPT has a term which converges to the AO theory when the focused two macromolecules are extremely larger than the other macromolecules. The XSPT can be used as the more precise version of the AO theory when the size ratio between the focused macromolecule and the surrounding macromolecule in the solution is nearly equal. We studied two points in this work. I) we verified the basic assumption used in the XSPT that topological elements which determine the analytical expression of the excluded volume are almost unchanged through the scaling down of the solute molecule in the thought experiment. II) the association of the actin monomers and the actin trimers are studied.

\section{XSPT}

Solvation free energy of the solute molecule is divided into three steps in the thought experiment in the XSPT. (I) the work required for cavity formation in the solvent, $g_{C}$, (II) contribution from the Lennard-Jones type attraction between the solute and the solvent, $g_{I}$, (III) electrostatic contribution, Born energy, $g_{B}$. The work for the cavity formation in the solvent, $g_{C}$, can be given by calculating the work to make the cavity in the hard sphere solvent. The solvation free energy in the dilute solution, $g_{C}$, is $[7,8]$

$$
\begin{aligned}
& g_{C}=A+B+\frac{1}{2} C+P V_{c}(1) \\
& A=-k_{B} T \ln \left(1-\rho V_{c}(0)\right), \quad B=k_{B} T \frac{1}{1-\rho V_{c}(0)} \rho\left(\frac{\partial V_{c}}{\partial \lambda}\right)_{\lambda=0}, \\
& C=k_{B} T \frac{1}{1-\rho V_{c}(0)} \rho\left(\frac{\partial^{2} V_{c}}{\partial \lambda^{2}}\right)_{\lambda=0}+k_{B} T \frac{1}{\left(1-\rho V_{c}(0)\right)^{2}} \rho^{2}\left(\left(\frac{\partial V_{c}}{\partial \rho}\right)_{\lambda=0}\right)^{2},
\end{aligned}
$$




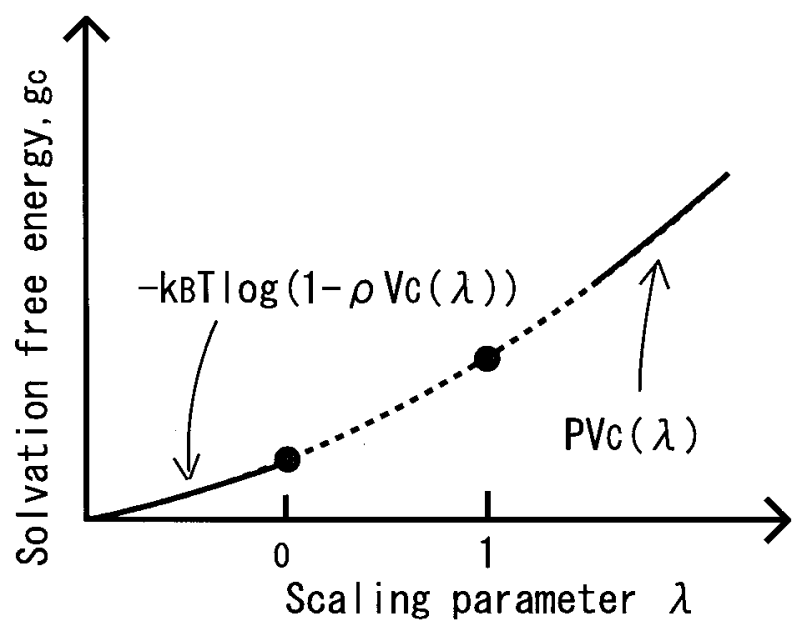

Figure 1. Schematic drawing of the $\lambda$ dependence of the solvation free energy, $g_{C}$. The symbols $\rho$ and $V_{c}(\lambda)$ are the number density of the solvent and the excluded volume of the $\lambda$-fold scaled solute molecule, $P$ is the pressure of the system.

where $V_{c}(\lambda)$ is the excluded volume of the $\lambda$-fold scaled solute, $P$ is the pressure of the system, $k_{B}$ is the Boltzmann constant, $T$ is temperature, $\rho$ is the number density of the solvent. The value of $V_{c}(0)$ is equal to the volume of the solvent molecule. The Equation (1) uses charging formula in the statistical mechanics by scaling the solute molecule which result the changes of the shape of the corresponding cavity in the hard sphere solvent. When $\lambda$ is less than zero, it is known that exact statistical mechanical equation to calculate the work required to make the cavity in the hard sphere solvent exists [6]. If the solute has macroscopic size, the thermodynamic equation can be used to calculate the work for the cavity formation. The scaled particle theory fulfills the both microscopic and macroscopic conditions (Figure 1). The XSPT can be regarded as the interpolation method of the microscopic and macroscopic exact equations. Then Equation (1) implicitly assumes the existence of the single third polynomial function to approximate the excluded volume.

\section{Assumption Used in the Calculation of the Work Required to Make the Cavity in the XSPT}

The topology obtained at $\lambda=1$ has been used in the calculation of the derivatives of the excluded volume in XSPT. We verified the basic assumption used in the XSPT that topological elements [10] which determine the analytical expression of the excluded volume are almost unchanged through the scaling down of the solute molecule in the thought experiment.

In this work, the radius of the atom in the solute molecule has the same value, $0.19 \mathrm{~nm}$, and the solvent molecule, water molecule, is regarded as a single sphere which has the radius of $0.1375 \mathrm{~nm}$. The solute molecule is hypothetically $\lambda$-fold 

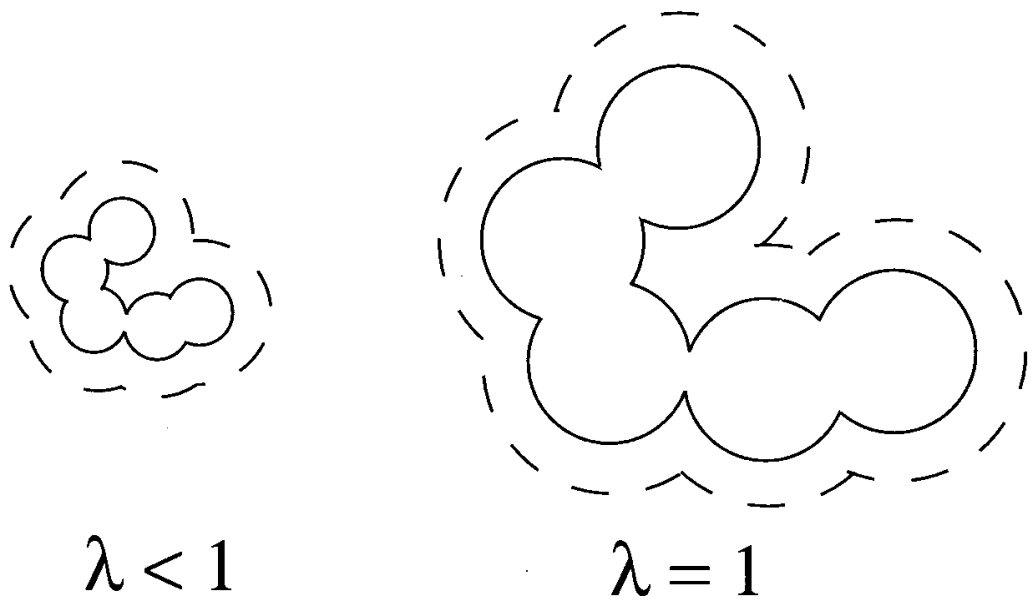

Figure 2. The excluded volume of the $\lambda$-fold scaled fused hard spheres. The dashed line represents the excluded volume of the fused hard spheres. The topology of the excluded volume at $\lambda<1$ is different from that at $\lambda=1$.

scaled with the solvent molecules having fixed radii in the XSPT. The shape of the excluded volume is the fused spheres whose sphere radius has the value of atom radius plus solvent molecule radius. The shape of the excluded volume of $\lambda$-fold scaled solute molecule is not similar to the shape of the solute molecule (Figure 2). The analytical expression of the excluded volume function at any $\lambda$ value is required in the XSPT. The analytical derivatives of the excluded volume by the $\lambda$ are also required. After the topology of the excluded volume is known, the analytical derivatives of the excluded volume, which is the fused spheres, is obtained by using the analytical expression of volume of the fused spheres developed by us [10]. During the scaling process of the solute molecule, the topology of the excluded volume is changed depending on the $\lambda$ value, in spite of the $\lambda$-fold solute molecule has the same topology at any $\lambda$ value. In the particular case, when the $\lambda$ decreases to zero, the shape of the excluded volume approaches a single sphere but the topology of the excluded volume becomes complicated. It is required to obtain the exact information of the topology of the excluded volume at any $\lambda$ value. The topology search like this case is a difficult problem for conventional Neumann type computers.

\section{4. $\alpha$-Shape Method}

In the computational geometry, $\alpha$-shape method [11] is the method to exactly and quickly calculate the table of the topology of the fused spheres as the function of the radius of the sphere, $\alpha$, keeping the configuration of the centers of the spheres fixed (Figure 3) [12]. The $\alpha$ values at which the topology is changed are calculated by using the Voronoi polyhedron in the $\alpha$-shape method. The shape of the fused 


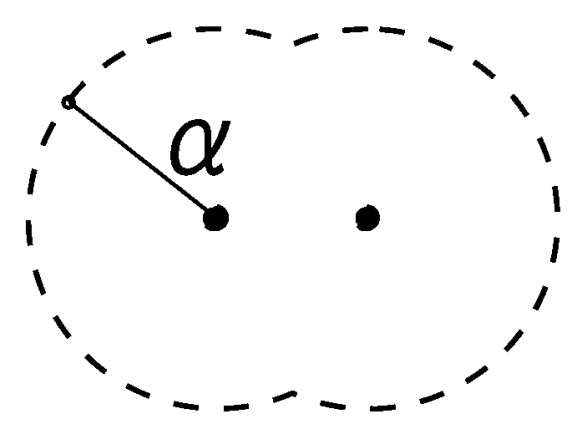

Figure 3. The $\alpha$ value in the $\alpha$-shape. The dashed line represents the $\alpha$-shape of the fused spheres (dumbbell). The radius of the sphere in the $\alpha$ shape is $\alpha$.

sphere is changed depending on the $\alpha$ value. The shape of the fused sphere approaches a single sphere when the $\alpha$ value approaches infinite. The fused spheres corresponding to the excluded volume in the XSPT becomes to be similar as the one in the $\alpha$-shape method when the following condition is satisfied.

$$
\alpha=\frac{r_{v}}{\lambda}+r_{s}
$$

where $r_{s}$ and $r_{v}$ represent the radius of the solute atom and the solvent molecule, respectively. The topology is unchanged in the similar figures. The $\alpha$-shape method can be used for the topology search of the excluded volume in the XSPT. The values of the $\lambda$ where the topology of the excluded volume is changed are obtained together with the each topological information of the excluded volume. The $\alpha$ shape method is necessary for the solute molecules having the large number of atoms, e.g. biomolecules, in the XSPT.

\section{The Potential of Mean Force}

The potential of mean force, $U(a)$, of the attractive force between two macromolecules at distance $a$ through the excluded volume effect is calculated from the work, $W(a)$, required to make the cavity in the solution which corresponds to the excluded volume of the two macromolecules (Figure 4).

$$
U(a)=W(a)-W(\infty)=W(a)-2 W(0)
$$

This system can be modeled by following the traditional polymer theory as a hard sphere system composed of two hard spheres whose positions are suspended and the surrounding hard sphere liquid. In this work, the interaction through the excluded volume effect between the macromolecules are calculated by using the solution theory, XSPT. The work required to make the cavity of the two macromolecules, $W(a)$, at the packing fraction $\eta$ is

$$
W(a)=A+B+\frac{1}{2} C+P V_{c}(1), \eta=\frac{4}{3} \pi r_{v}^{3} \rho
$$




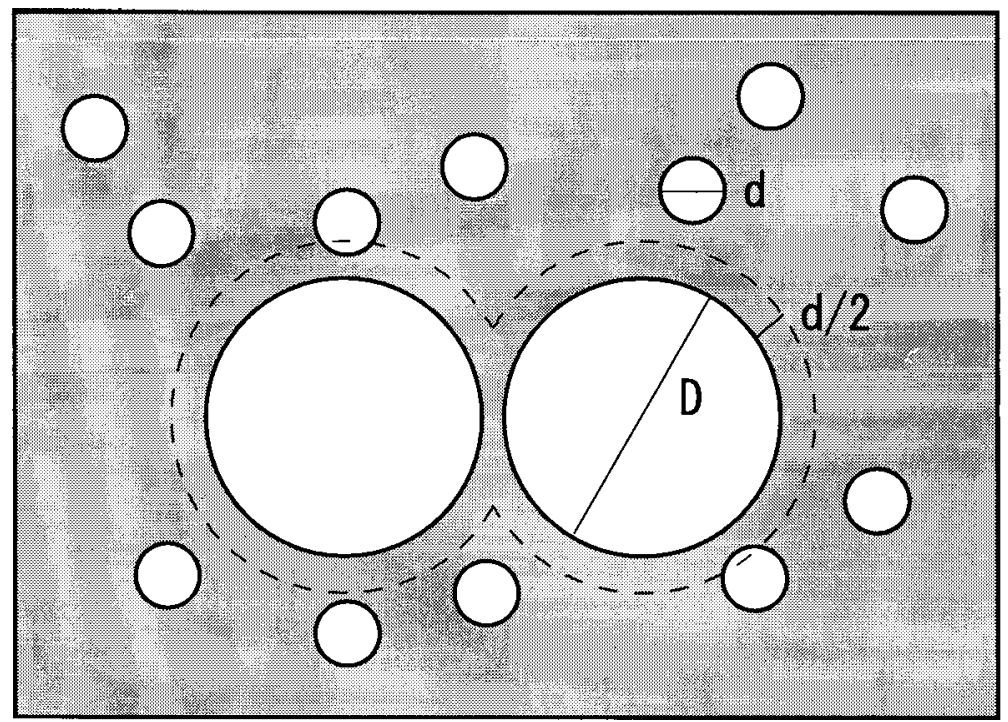

Figure 4. Two macromolecules interacting through the excluded volume effect. The focused two macromolecules having the diameter of $D$ are suspended in the solution of macromolecules having the diameter of $d$. The dashed line represents the excluded volume of the focused two macromolecules against the surrounding macromolecules.

$$
\frac{P}{\rho k_{B} T}=\frac{1+\eta+\eta^{2}}{(1-\eta)^{3}}
$$

where $P$ is the osmotic pressure and calculated by using the equation of state derived by SPT for the hard sphere liquid [6], $A, B$, and $C$ are the same expression in Equation (1). But the $V_{c}(\lambda)$ is not the same as the one in Equation (1). The $V_{c}(\lambda)$ in Equation (1) is the excluded volume of the solute molecule against the solvent molecule in the dilute solution, the $V_{c}(\lambda)$ in Equation (4) is the excluded volume of the 'two' macromolecules against the surrounding macromolecules. The term $C$ in Equation (4) is roughly proportional to the surface area of the cavity made by two macromolecules. The term, $P V_{c}(1)$, in Equation (4) converges to the AO theory when the focused two macromolecules are extremely larger than the other macromolecules.

We used two protein molecules, one is avian pancreatic polypeptide having 26 residues and another is G-actin having 373 residues. The protein data bank ID [13] of the 3-D structures of avian pancreatic polypeptide and the G-actin used in the calculation are 1PPT [14] and 1ATN (chain A) [15] respectively. The trimer 3-D model structure (Lorenz model [1]) of the F-actin is used in the calculation. The trimer 3-D model structure is called as Lorenz 3 in this paper. The monomer form of the model structure, called Lorenz 1, is taken from the Lorenz 3. The G-actin and Lorenz 1 have the hetero atoms, ATP and ADP respectively. A 'modified Lorenz 1' has the same number of residues as G-actin by removing C-terminal three residues. 


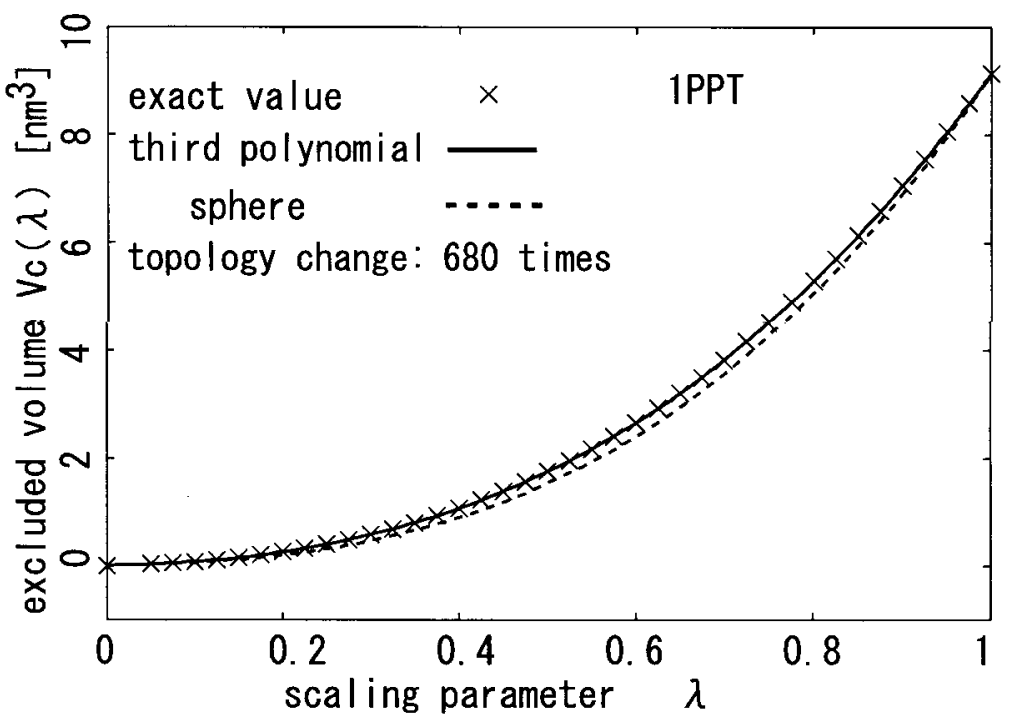

Figure 5. Excluded volume of the Avian pancreatic polypeptide. The cross, solid line, and dashed line represent the exact value of the excluded volume, the approximated third polynomial value of the excluded volume, and the value of the single sphere which has the same value as the exact one at $\lambda=1$, respectively.

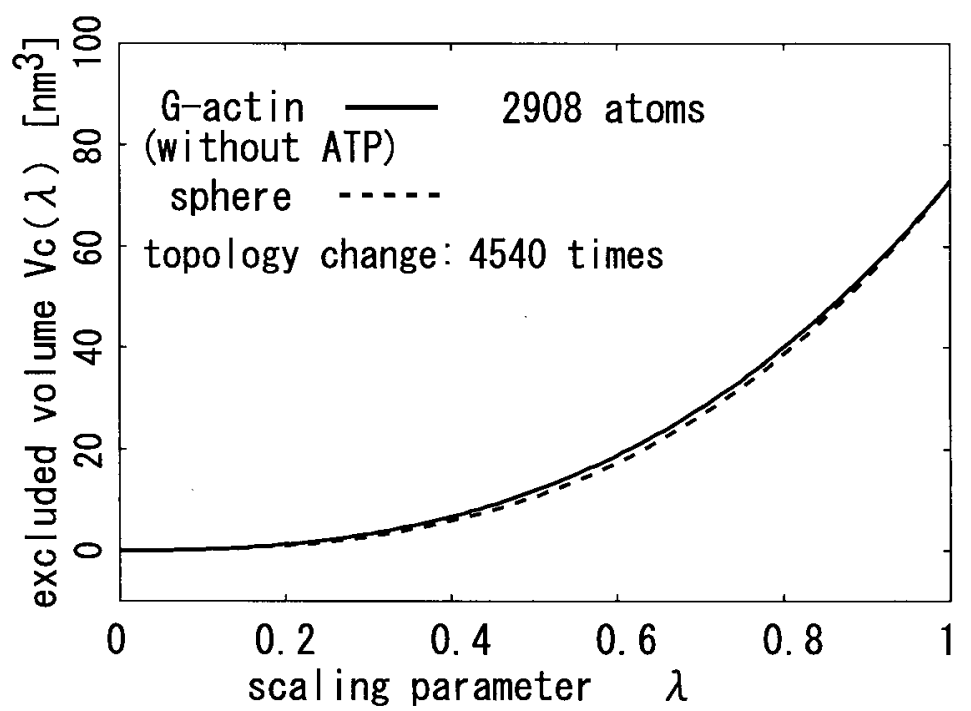

Figure 6. Excluded volume of the monomeric G-actin molecule. The solid line and dashed line represent the approximated third polynomial value of the excluded volume, and the value of the single sphere which has the same value as the exact one at $\lambda=1$, respectively. 


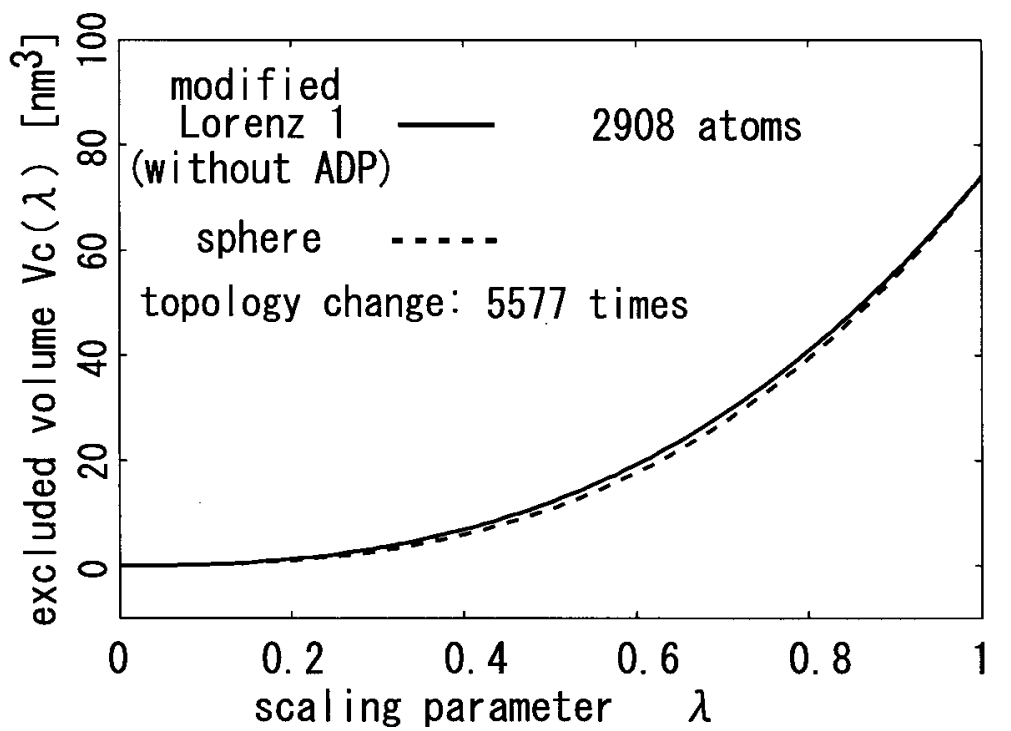

Figure 7. Excluded volume of the modified Lorenz 1. The solid line and dashed line represent the approximated third polynomial value of the excluded volume, and the value of the single sphere which has the same value as the exact one at $\lambda=1$, respectively.

Table I. The excluded volumes and corresponding spherical radii

\begin{tabular}{|c|c|c|c|c|}
\hline & Topology change & $V_{c}(1)\left[\mathrm{nm}^{3}\right.$ & cal radius $[\mathrm{nm}]$ & $\mathrm{ASA}\left[\mathrm{nm}^{2}\right]$ \\
\hline 1PPT & 680 & 9.14007 & 1.1595 & 34.3917 \\
\hline G-actin without ATP & 4540 & 72.76704 & 2.4514 & 165.3733 \\
\hline modified Lorenz 1 & 5461 & 74.34961 & 2.4710 & 174.8621 \\
\hline modified Lorenz 1 without ADP & 5577 & 74.10972 & 2.4682 & 176.8723 \\
\hline Lorenz 1 & 5489 & 74.87227 & 2.4771 & 175.4681 \\
\hline Lorenz 1 without ADP & 5605 & 74.63237 & 2.4743 & 177.4783 \\
\hline Lorenz 3 & 16762 & 220.0763 & 3.6079 & 473.4202 \\
\hline Lorenz 3 without ADP & 17111 & 219.3566 & 3.6038 & 479.4505 \\
\hline
\end{tabular}

\section{Results and Discussion}

The excluded volume of avian pancreatic polypeptide which is hypothetically scaled is exactly calculated. Exact analytical expression of the excluded volume is not the third polynomial of the scaling parameter $\lambda$ [10]. The topology of the protein is changed 680 times during the scaling and the analytical expression is changed at each topology change. But the excluded volume $\left(\mathrm{nm}^{3}\right)$ can be approximated as the single function of the third polynomial by the method of least squares,

$$
V_{c}(\lambda)=4.80181 \lambda^{3}+4.04587 \lambda^{2}+0.285835 \lambda+0.01089 .
$$


Table II. The values of the potential of the attraction between actin molecules through the excluded volume effect

\begin{tabular}{lccc}
\hline focused + surrounding molecules & $\frac{U(D)}{k_{B} T}$ & $\frac{U(D+d)}{k_{B} T}$ & $\frac{U(D)}{k_{B} T}-\frac{U(D+d)}{k_{B} T}$ \\
\hline G-actin + G-actin (XSPT) & -0.02509 & 0.00035 & -0.02544 \\
(AO theory) & -0.02500 & 0 & -0.02500 \\
Lorenz 1 + Lorenz 1 (XSPT) & -0.02509 & 0.00035 & -0.02544 \\
(AO theory) & -0.02500 & 0 & -0.02500 \\
Lorenz 3 + G-actin (XSPT) & -0.03170 & 0.00058 & -0.03228 \\
(AO theory) & -0.03167 & 0 & -0.03167 \\
Lorenz 3 + Lorenz 1 (XSPT) & -0.03152 & 0.00057 & -0.03209 \\
(AO theory) & -0.03149 & 0 & -0.03149 \\
\hline
\end{tabular}

$k_{B}$ : Boltzmann constant, $T$ : temperature.

The standard deviations are $\pm 0.2767 \%, \pm 0.4938 \%$, and $\pm 3.582 \%$ respectively. In Figure 5, the dashed line is the volume of the single sphere which has the same volume as the excluded volume of the protein when $\lambda$ is equal 1 . The difference between the excluded volumes of the protein and the single sphere shows the effect of the non-spherical shape of the protein. The excluded volumes of the two different actin structures are calculated. The actin monomers take the G-actin structure in the solution and the Lorenz 1 structure in the filament, F-actin. The excluded volume $\left(\mathrm{nm}^{3}\right)$ of the G-actin is approximated as,

$$
V_{c}(\lambda)=50.7710 \lambda^{3}+22.1181 \lambda^{2}-0.0873322 \lambda+0.01089
$$

The standard deviations are $\pm 0.2726 \%, \pm 0.8562 \%, \pm 70.83 \%$ respectively. The excluded volume $\left(\mathrm{nm}^{3}\right)$ of the modified Lorenz 1 is approximated as,

$$
V_{c}(\lambda)=50.6902 \lambda^{3}+23.7339 \lambda^{2}-0.264775 \lambda+0.01089 .
$$

The standard deviations are $\pm 0.3374 \%, \pm 0.9862 \%, \pm 28.87 \%$ respectively. Figure 6 and Figure 7 show the plot of the excluded volume of the actin molecules against the scaling parameter $\lambda$. The exact values of the excluded volume are not plotted in Figure 6 and Figure 7 because the values are almost the same as those of the approximated third polynomials. The approximate third polynomial of $\lambda$ gives good agreement with the exact values of the excluded volume of the proteins because the frequent topology changes seem to average the effect of the individual topology change. Results of the computational geometry method ( $\alpha$-shape method) showed that the assumption, we have used in XSPT, is valid even in the case of the actin molecules. Table I shows the excluded volumes and corresponding spherical solute radii. The $V_{c}(1)$ is the real excluded volume at $\lambda=1$. The values of accessible surface area (ASA) of the molecules are also listed.

The values of the potential of the long-range attraction between the actin molecules through the excluded volume effect is calculated by using the XSPT and 
Asakura-Oosawa theory (AO theory) and listed in Table II. The shape of the all actin molecules is approximated as a single sphere having the effective radius listed in Table I. The number density is determined from the packing fraction of 0.01 [2]. The absolute values of the potential of the attraction between the actin molecules are small at $\eta=0.01$ in this study because the size of the focused and surrounding actin molecules is nearly equal. The effect of the terms except for $P V_{c}(1)$ in Equation (4) is negligible at low number density of the surrounding macromolecules. When the direct attraction between macromolecules like Lennard-Jones type interaction decreases the osmotic pressure, the term which is roughly proportional to the accessible surface area becomes to be important. The conformational change from G-actin in the solution to Lorenz 1 in the F-actin does not affect the attraction through the excluded volume in our results. It is worth to search the condition where the effect of the conformational change of the actin molecule on the attraction is large in the future study. These findings have the suggestion to the future study of the association mechanism of the macromolecules.

\section{Acknowledgements}

This work was supported, in part, from the Grant-in-Aid for Encouragement of Young Scientists (11780473) and the Research for the Future Program (JSPSRFTF98P01101) of the Japan Society for the Promotion of Science, and from the Ministry of Education, Science, Sports and Culture.

\section{References}

1. Lorenz, M., Popp, D. and Holmes, K.C.: Refinement of the F-Actin Model against X-ray Fiber Diffraction Data by the Use of a Directed Mutation Algorithm, J. Mol. Biol. 234 (1993), 826836.

2. Asakura, S. and Oosawa, F.: Interaction between particles suspended in solutions of macromolecules, J. Poly. Sci. 33 (1958), 183-192.

3. Gast, E.P., Hall, C.K. and Bussel, W.B.: Polymer-induced phase separations in nonaqueous colloidal suspensions, J. Colloid Interface Sci. 96 (1983), 251-267.

4. Gast, A.P., Russel, W.B. and Hall, C.K.: An experimental and theoretical study of phase transitions in the polystyrene latex and hydroxyethylcellulose system, J. Colloid Interface Sci. 109 (1986), 161-171.

5. Patel, P.D. and Russel, W.B.: An experimental study of aqueous colloidal suspensions containing dissolved polymer, J. Colloid Interface Sci. 131 (1989), 201-210.

6. Reiss, H., Fish, H.L. and Lebowitz, J.L.: Statistical mechanics of rigid spheres, J. Chem. Phys. 31 (1959), 369-380.

7. Irisa, M., Nagayama, K. and Hirata, F.: Extended scaled particle theory for dilute solutions of arbitrary shaped solutes. An application to solvation free energies of hydrocarbons, Chem. Phys. Lett. 207 (1993), 430-435.

8. Irisa, M., Takahashi, T., Nagayama, K. and Hirata, F.: Solvation free energies of non-polar and polar solutes reproduced by a combination of extended scaled particle theory and the PoissonBoltzmann equation, Mol. Phys. 85 (1985), 1227-1238. 
9. Irisa, M., Takahashi, T., Hirata, F. and Yanagida, T.: Solvation Free Energy of Protein Reproduced by the Combination of the Extended Scaled Particle Theory and the Poisson-Boltzmann Equation, J. Mol. Liquids 65/66 (1995), 381-384.

10. Irisa, M.: An elegant algorithm of the analytical calculation for the volume of fused spheres with different radii, Comp. Phys. Comm. 98 (1996), 317-338.

11. Edelsbrunner, H., Facello, M., Fu, P. and Liang, J.: Measuring proteins and voids in proteins, Proceedings of the 28th Annual Hawaii International Conference on System Science (1995), 256-264.

12. http://www.alphashapes.org/alpha/index.html

13. Berman, H.M., Westbrook, J., Feng, Z., Gilliland, G., Bhat, T.N., Weissig, H., Shindyalov, I.N. and Bourne, P.E.: The Protein Data Bank, Nucl. Acids Res. 28 (2000), 235-242.

14. Glover, I., Haneeef, I., Pitts, J., Wood, S., Moss, D., Tickle, I. and Blundell, T.: Conformational Flexibility in a Small Globular Hormone: X-Ray Analysis of Avian Pancreatic Polypeptide at 0.98 - A Resolution, Biopolymers 22 (1983), 293-304.

15. Kabsh, W., Mannherz, H.G., Suck, D., Pai, E.F. and Holmes, K.C.: Atomic structure of the actin: DNase I complex, Nature 347 (1990), 37-44. 
